\title{
Editorial: Current research and emerging directions on the cognitive and neural organization of speech processing
}

\author{
Patti Adank ${ }^{1 *}$, Carolyn McGettigan ${ }^{2}$ and Sonja A. E. Kotz ${ }^{3,4}$ \\ 'Division of Psychology and Language Sciences, Speech, Hearing and Phonetic Sciences, University College London, \\ London, UK, ${ }^{2}$ Department of Psychology, Royal Holloway University of London, Egham, UK, ${ }^{3}$ Max Planck Institute Leipzig, \\ Leipzig, Germany, ${ }^{4}$ School of Psychological Sciences, University of Manchester, Manchester, UK
}

Keywords: speech perception, speech production, functional magnetic resonance imaging (fMRI), magnetoencephalography (MEG), electroencephalography, transcranial magnetic stimulation (TMS)

This Research Topic consists of 14 manuscripts discussing the cognitive and neural organization of speech processing. The contributions are grouped around four themes: (1) Spoken language comprehension under difficult listening conditions; (2) Sub-lexical processing; (3) Sensorimotor processing of speech; (4) Speech production.

Seven papers addressed speech perception under challenging listening conditions. Van Engen and Peelle (2014) discuss the effects of processing speech in an unfamiliar regional or foreign accent. They argue that, as perceiving accented speech incurs a processing cost, just like other types of distortions such as background noise, it should also be regarded as representing a challenging listening condition. Neger et al. (2014) focused on plasticity of speech processing in statistical and perceptual learning tasks in aging. They conclude that perceptual and statistical learning share mechanisms of implicit regularity detection, but that the ability to detect statistical regularities is impaired in older adults for fast visual sequences. Dekerle et al. (2014) examined whether speech perception in a multi-speaker background relies on semantic interference between the background and target speaker using a semantic priming paradigm in three experiments. Their results indicate that higher-level linguistic processes such as semantic priming may not be as automatic as commonly thought but are subjected to the limits of cognitive resources such as working memory and attention. Yi et al. (2014) evaluate how processing of foreign-accented speech relates to social cognition. It was concluded that foreign-accented speech perception engages greater activation of neural systems underlying speech perception, and that implicit Asian-foreign association is related to with decreased neural efficiency in early spectrotemporal processing. Vitello et al. (2014) used fMRI to address the question of how semantic ambiguities are resolved during speech comprehension.

Strauß et al. (2014) examined through literature review whether neural oscillations in the alpha

Received: 16 April 2015 Accepted: 12 May 2015 Published: 27 May 2015

Citation: Adank P, McGettigan C and Kotz SAE (2015) Editorial: Current research and emerging directions on the cognitive and neural organization of speech processing.

Front. Hum. Neurosci. 9:305. doi: 10.3389/fnhum.2015.00305 frequency range $(\sim 10 \mathrm{~Hz})$ act as a neural mechanism to selectively inhibit the processing of noise to improve auditory selective attention to task-relevant speech signals. Ding and Simon (2014) discuss whether cortical entrained activity is related more closely to speech perception or to auditory encoding that is not specific to speech, by reviewing evidence regarding various hypotheses about the functional roles of cortical entrainment to speech.

Three papers focused on perception of speech at sub-lexical levels. Deschamps and Tremblay (2014) studied perception of sub-lexical information by examining the neural bases of processing of simple syllables and more complex syllabic structures using fMRI, while Yu et al. (2014) used MEG to study the neural processing of disgust in anterior insula by presenting listeners with syllables with differed intended emotional meanings. Finally Chen et al. (2014) investigated processing of 
acoustic and phonological information in lexical tones in Mandarin Chinese using EEG.

Two papers addressed sensorimotor processing of speech. Komeilipoor et al. (2014) report higher motor excitability as measured using Transcranial Magnetic Stimulation (TMS) in the tongue area during the presentation of meaningful gestures (noun-associated). Sowman et al. (2014) demonstrate that appropriately timed TMS to the hand area, paired with auditorily mediated excitation of the motor cortex, induces an enhancement of motor cortex excitability that lasts beyond the time of stimulation.

Two papers focused on speech production. Etchell et al. (2014) provide a review of the stuttering literature and HernandezPavon et al. (2014) present a neuronavigated TMS study

\section{References}

Chen, C., Lee, Y., and Cheng, Y. (2014). Anterior insular cortex activity to emotional salience of voices in a passive oddball paradigm. Front. Hum. Neurosci. 8:743. doi: 10.3389/fnhum.2014.00743

Dekerle, M., Boulenger, V., Hoen, M., and Meunier, F. (2014). Multi-talker background and semantic priming effect. Front. Hum. Neurosci. 8:878. doi: 10.3389 /fnhum. 2014.00878

Deschamps, I., and Tremblay, F. (2014). Sequencing at the syllabic and suprasyllabic levels during speech perception: an fMRI study. Front. Hum. Neurosci. 8:492. doi: 10.3389/fnhum.2014.00492

Ding, N., and Simon, J. Z. (2014). Cortical entrainment to continuous speech: functional roles and interpretations. Front. Hum. Neurosci. 8:311. doi: 10.3389/fnhum.2014.00311

Etchell, A. C., Johnson, B. W., and Sowman, P. F. (2014). Behavioral and multimodal neuroimaging evidence for a deficit in brain timing networks in stuttering: A hypothesis and theory. Front. Hum. Neurosci. 8:467. doi: 10.3389/fnhum.2014.00467

Hernandez-Pavon, J. C., Mäkelä, N., Lehtinen, H., Lioumis, P., and Mäkelä, J. (2014). Effects of navigated TMS on object and action naming. Front. Hum. Neurosci. 8:660. doi: 10.3389/fnhum.2014.00660

Komeilipoor, N., Vicario, C. M., Daffertshofer, A., and Cerari, P. (2014). Talking hands: tongue motor excitability during observation of hand gestures associated with words. Front. Hum. Neurosci. 8:767. doi: 10.3389/fnhum.2014.00767

Neger, T. M., Rietveld, T., and Janse, E. (2014). Relationship between perceptual learning in speech and statistical learning in younger and older adults. Front. Hum. Neurosci. 8:628. doi: 10.3389/fnhum.2014.00628

Sowman, P. F., Rasmussen, J., Dueholm, S., and Mrachacz-Kersting, N. (2014). Induction of plasticity in the human motor cortex by pairing exploring the neural locus of aspects of picture naming in healthy participants.

This Frontiers Research Topic allows new insights into the neurobiology of speech perception and production, and demonstrates how the field of speech science is now addressing issues at its very core. We believe that the future of the research in the field lies in the effective combination of research methods, e.g., EEG and TMS, or fMRI and EEG, as research will benefit from the strengths of each method. In conclusion, this Research Topic consists of 14 excellent contributions, and we are convinced the Topic will provide readers with novel ideas for future studies that will elucidate the cognitive and neural architecture of speech processing.

an auditory stimulus with TMS. Front. Hum. Neurosci. 8:398. doi: 10.3389/fnhum.2014.00398

Strauß, A., Wöstmann, M., and Obleser, J. (2014). Cortical alpha oscillations as a tool for auditory selective inhibition. Front. Hum. Neurosci. 8:350. doi: 10.3389/fnhum.2014.00350

Van Engen, K. J., and Peelle, J. E. (2014). Listening effort and accented speech. Front. Hum. Neurosci. 8:577. doi: 10.3389/fnhum.2014 00577

Vitello, S., Warren, J. E., Devlin, J. T., and Rodd, J. M. (2014). Roles of frontal and temporal regions in reinterpreting semantically ambiguous sentences. Front. Hum. Neurosci. 8:530. doi: 10.3389/fnhum.2014.00530

Yi, H., Smiljanic, R., and Chandrasekaran, B. (2014). The neural processing of foreign-accented speech and its relationship to listener bias. Front. Hum. Neurosci. 8:768. doi: 10.3389/fnhum.2014.00768

Yu, K., Wang, R., Li, L., and Li, P. (2014). Processing of acoustic and phonological information of lexical tones in mandarin chinese revealed by mismatch negativity. Front. Hum. Neurosci. 8:729. doi: 10.3389/fnhum.2014 00729

Conflict of Interest Statement: The authors declare that the research was conducted in the absence of any commercial or financial relationships that could be construed as a potential conflict of interest.

Copyright (c) 2015 Adank, McGettigan and Kotz. This is an open-access article distributed under the terms of the Creative Commons Attribution License (CC BY). The use, distribution or reproduction in other forums is permitted, provided the original author(s) or licensor are credited and that the original publication in this journal is cited, in accordance with accepted academic practice. No use, distribution or reproduction is permitted which does not comply with these terms. 